\title{
Diffusion-weighted imaging in identifying breast cancer pathological response to neoadjuvant chemotherapy: A meta- analysis
}

\author{
Wei Chu ${ }^{1, *}$, Weiwei Jin ${ }^{2, *}$, Daihong Liu ${ }^{3}$, Jian Wang ${ }^{3}$, Chengjun Geng ${ }^{4}$, Lihua Chen ${ }^{4}$ \\ and Xuequan Huang ${ }^{3}$ \\ ${ }^{1}$ Department of Radiology, Wuxi Huishan District People's Hospital, Jiangsu Province, 214187, China \\ ${ }^{2}$ Department of Radiology, Wuxi Second Traditional Chinese Medicine Hospital, Jiangsu Province, 214121, China \\ ${ }^{3}$ Department of Radiology, Southwest Hospital, Third Military Medical University, Chongqing, 400038, China \\ ${ }^{4}$ Department of Radiology, PLA No.101 Hospital, Wuxi, Jiangsu Province, 214044, China \\ *These authors contributed equally to this work \\ Correspondence to: Xuequan Huang, email: hxuequan@163.com \\ Lihua Chen, email: clhaaa2002@163.com \\ Keywords: magnetic resonance imaging; diffusion-weighted imaging; neoadjuvant chemotherapy; breast cancer; \\ meta-analysis \\ Received: October 27, 2017 \\ Accepted: December 01, 2017 \\ Published: December 11, 2017 \\ Copyright: Chu et al. This is an open-access article distributed under the terms of the Creative Commons Attribution License 3.0 (CC BY \\ 3.0), which permits unrestricted use, distribution, and reproduction in any medium, provided the original author and source are credited.
}

\section{ABSTRACT}

Background: Diffusion-weighted imaging (DWI) is increasingly used to identify pathological complete responses (pCRs) to neoadjuvant chemotherapy (NAC) in breast cancer. The aim of the present study was to assess the utility of DWI using a pooled analysis.

Materials and Methods: Literature databases were searched prior to July 2017. Fifteen studies with a total of 1181 patients were included. The data were extracted to perform pooled analysis, heterogeneity testing, threshold effect testing, sensitivity analysis, publication bias analysis and subgroup analyses.

Result: The methodological quality was moderate. Remarkable heterogeneity was detected, primarily due to a threshold effect. The pooled weighted values were a sensitivity of 0.88 (95\% confidence interval (CI): $0.81,0.92)$, a specificity of 0.79 (95\% CI: $0.70,0.86$ ), a positive likelihood ratio of 4.1 (95\% CI: $2.9,5.9$ ), a negative likelihood ratio of 0.16 (95\% CI: $0.10,0.24)$, and a diagnostic odds ratio of 26 (95\% CI: 15, 46). The area under the receiver operator characteristic curve was 0.91 ( $95 \% \mathrm{CI}: 0.88,0.93)$. In the subgroup analysis, the pooled specificity of change in the apparent diffusion coefficient (ADC) subgroup was higher than that in the pretreatment ADC subgroup (0.80 [95\% CI: $0.71,087]$ vs. 0.63 [95\% CI: $0.52,0.73$, $P=0.027$ ).

Conclusions: DWI may be an accurate and nonradioactive imaging technique for identifying PCRs to NAC in breast cancer. Nonetheless, there are a variety of issues when assessing DWI techniques for estimating breast cancer responses to NAC, and large scale and well-designed clinical trials are needed to assess the technique's diagnostic value.

\section{INTRODUCTION}

Neoadjuvant chemotherapy (NAC) has been used as a standard treatment for both initially operable and initially inoperable locally advanced breast cancer [1] Patients who achieve a pathologic complete response (pCR; defined as no residual tumour or a minimal residual tumour on histologic analysis) demonstrate significantly 
longer disease-free and overall survival rates [2]. The early prediction of outcome and identification of the $\mathrm{pCR}$ to NAC are important for individualised therapies and avoiding the use of additional toxic therapies and provide a greater chance of achieving a pCR $[3,4]$.

Determining how to predict the pCR to NAC accurately remains a challenging clinical problem with no consensus approach. Based on their quantitative and noninvasive characteristics, several imaging tools, such as magnetic resonance imaging (MRI), mammography, and ultrasound, are used to monitor tumour size change after NAC [5]. However, these imaging techniques, which focus on monitoring changes in morphological features, are unable to distinguish potential residual cancer from fibrotic scar tissue in a stable tumour [6]. These limitations have led many researchers to explore other functional techniques, such as positron emission tomography, quantitative perfusion MRI, magnetic resonance spectroscopy and diffusion-weighted imaging (DWI). Based on the diffusion of water molecules through tumour tissue, DWI is a new means of predicting tumour responses to treatment [7]. The Brownian motion of water molecules in cancer is restricted, which results in a decreased apparent diffusion coefficient (ADC) value. Previous studies [8-10] have shown that the ADC is highly negatively correlated with tumour cellularity and could be used to estimate the tumour pathological response to therapy.

Many studies [11-25] have reported the accuracy of DWI in predicting pathological responses to NAC in breast cancer against a histopathologic reference standard. However, the findings of these studies have been incongruent, as different DWI techniques have been used, and most of the sample sizes have been small. Therefore, we conducted a meta-analysis to assess the diagnostic performance of DWI for monitoring pathological responses to NAC in breast cancer.

\section{RESULTS}

Medical and scientific literature databases were searched and reference lists were cross-checked for original articles published prior to July 2017 (Figure 1), 166 articles were found in the primary result. There were 15 studies [11-25] with a total of 1181 breast cancer patients that met the inclusion criteria for quantitative synthesis.

The data extracted from these included studies are summarised in Tables 1, 2 and Supplementary Table 1. The mean number of patients per study was 74.6 (range 20-195), and the mean age of the patients in each study was 48.7 years (range 23-83). A variety of NAC regimens, definitions of pCR, MRI devices, DWI methods of measurement, analyses, and cutoff values were observed. Of the 15 studies, 9 studies [11-16, 22-24] evaluated both DWI and conventional contrast-enhanced MRI (CE-MRI) techniques in the same cohort, 6 studies $[14,16-18,23$, $25]$ were performed using $3 \mathrm{~T}$ scanners, 5 studies [12$14,22,24]$ were performed with a $b$ value $\geq 1000 \mathrm{~s} / \mathrm{m}^{2}$, and 2 studies $[14,25]$ were performed using intravoxel incoherent motion (IVIM) models. There were 4 studies $[12,15,16,25]$ that enrolled patients prospectively, and 8 studies [11, 13, 17, 19-23] that enrolled patients retrospectively, while the remaining studies did not report this parameter. A total of 7 studies [11-13, 18, 19, 23, 24] used the change in ADC as a biomarker, while 3 studies $[15,21,25]$ used the pre-treatment ADC, 2 studies [17, 20] used the post-treatment ADC, and 3 studies [14, 16, 22] used all three conditions.

According to QUADAS-2 items, the quality assessment of 15 studies was moderate. Only 3 studies [11-13] set a pre-specified threshold. The results of the distribution are shown in Figure 2.

As there was notable heterogeneity in the present metaanalysis $\left(\mathrm{I}^{2}=93 \%, P<0.001\right)$, we used a random-effects coefficient binary regression model. The pooled weighted values for DWI were a SEN of 0.88 ( $95 \%$ confidence interval (CI): 0.81, 0. 92), a SPE of 0.79 (95\% CI: $0.70,0.86)$, a positive likelihood ratio (PLR) of 4.1 (95\% CI: 2.9, 5.9), an negative likelihood ratio (NLR) of 0.16 (95\% CI: $0.10,0.24)$, a diagnostic odds ratio (DOR) of 26 (95\% CI: 15, 46), and an area under the ROC curve (AUC) of 0.91 (95\% CI: 0.88, 0.93). Forest plots and HSROC curves of the 15 studies are shown in Figures 3, 4, Supplementary Figures 1, and 2. After the sensitivity analysis, 3 studies $[17,22,24]$ were detected (Supplementary Figure 3). However, there was no effect on the results of the pooled weighted values when these studies were excluded. The proportion of heterogeneity likely due to a threshold effect was $95 \%$ in the accuracy estimates among individual studies. The results of meta-regression also indicated that $\mathrm{b}$ values, study design, MRI field strength, and DWI model were not strongly associated with accuracy.

The results of the subgroup analysis are presented in Table 3. In the subgroup analysis of b value, MRI field, study design and DWI model, no notable differences were observed. In the subgroup analysis of different biomarkers of DWI, the performance of the $\triangle \mathrm{ADC}$ subgroup was equivalent to that of the post-NAC ADC subgroup in assessing the pCR to NAC with comparable pooled sensitivity $(0.88$ [95\% CI: $0.75,0.94]$ vs. 0.91 [95\% CI: $0.78,0.96], \mathrm{P}=0.527$ ) and pooled specificity $(0.80$ [95\% CI: $0.71,087]$ vs. 0.78 [95\% CI: $0.58,0.90]$, $\mathrm{P}=0.398)$. However, the pooled specificity of the $\triangle \mathrm{ADC}$ subgroup was higher than that of the pre-NAC ADC subgroup (0.80 [95\% CI: $0.71,087]$ vs. 0.63 [95\% CI: $0.52,0.73], P=0.027$ ).

The results of the Deeks funnel plot asymmetry test showed no evidence of notable publication bias $(\mathrm{P}=0.50)$; see in Figure 5.

To compare the accuracy between DWI and CEMRI effectively, we performed a pooled analysis using head-to-head comparative diagnostic accuracy studies 
Table 1: Cohort and tumour characteristics of the included studies

\begin{tabular}{|c|c|c|c|c|c|}
\hline \multicolumn{2}{|c|}{ Variables } & Studies No. & Patients No. & Mean & Range \\
\hline \multicolumn{6}{|c|}{ Cohort characteristics } \\
\hline \multicolumn{2}{|l|}{ No., all tests } & 15 & 1081 & 74.6 & $20-225$ \\
\hline \multicolumn{2}{|l|}{ pCR } & 15 & 297 & $27.1 \%$ & $12.9 \%-85.0 \%$ \\
\hline \multicolumn{2}{|l|}{ non-pCR } & 15 & 783 & $72.9 \%$ & $15.0 \%-87.1 \%$ \\
\hline \multicolumn{2}{|l|}{ Age (years) } & 14 & 1011 & 48.7 & $23-83$ \\
\hline \multicolumn{6}{|c|}{ Tumour characteristics } \\
\hline \multirow[t]{3}{*}{ Stage } & II & 7 & 173 & $31.5 \%$ & $10.0 \%-63.3 \%$ \\
\hline & III & 8 & 355 & $64.5 \%$ & $36.7 \%-100 \%$ \\
\hline & IV & 1 & 22 & $18.4 \%$ & - \\
\hline \multirow[t]{4}{*}{ Histology } & IDC & 12 & 799 & $88.0 \%$ & $74.5 \%-97.2 \%$ \\
\hline & ILC & 10 & 85 & $9.8 \%$ & $3.3 \%-22.6 \%$ \\
\hline & $\mathrm{MC}$ & 3 & 6 & $3.1 \%$ & $2.9 \%-3.3 \%$ \\
\hline & Other & 8 & 33 & $4.2 \%$ & $1.1 \%-11.7 \%$ \\
\hline \multirow[t]{6}{*}{ Receptor } & $\operatorname{ER}(+)$ & 6 & 133 & $32.7 \%$ & $24.3 \%-46.9 \%$ \\
\hline & $\mathrm{PR}(+)$ & 5 & 134 & $32.8 \%$ & $25.0 \%-39.1 \%$ \\
\hline & HER-2 (+) & 11 & 185 & $21.0 \%$ & $6.3 \%-37.9 \%$ \\
\hline & LA & 4 & 109 & $26.5 \%$ & $12.5 \%-38.1 \%$ \\
\hline & LB & 4 & 106 & $41.9 \%$ & $25.6 \%-81.3 \%$ \\
\hline & $\mathrm{TN}$ & 7 & 138 & $19.5 \%$ & $7.4 \%-33.9 \%$ \\
\hline
\end{tabular}

Note: $\mathrm{ER}=$ oestrogen receptor; $\mathrm{PR}=$ progesterone receptor; HER-2 $=$ human epidermal growth factor receptor 2; IDC $=$ invasive ductal carcinoma; ILC = invasive lobular carcinoma; LA = luminal A; LB = luminal B; MC = mucinous carcinoma; $\mathrm{NAC}=$ neoadjuvant chemotherapy; $\mathrm{pCR}=$ pathologic complete response; $\mathrm{TN}$, triple negative.

(Table 4, Figure 6). The pooled weighted values for DWI were a SEN of 0.89 (95\% CI: $0.81,0.93)$, a SPE of 0.81 (95\% CI: $0.71,0.89)$, a DOR of 33.72 (95\% CI: 13.93, 81.59), and an AUC of 0.91 (95\% CI: $0.88,0.93)$. The pooled weighted values for CE-MRI were a SEN of 0.84 (95\% CI: $0.74,0.91)$, a SPE of 0.76 (95\% CI: 0.64, 0.85), a DOR of 16.57 (95\% CI: 9.80, 28.02), and an AUC of 0.88 (95\% CI: 0.84, 0.90).

\section{DISCUSSION}

Although breast MRI has been recommended as a clinical tool for NAC response evaluation for operable breast cancer, DWI has emerged as a potential imaging modality providing an early response biomarker based on ADC [26]. In this meta-analysis, we aimed to provide an overview of current strengths and weaknesses of DWI and to evaluate its accuracy for predicting the pCR to NAC in breast cancer using available data. The AUC of 15 studies concerning the new modality for estimating the pCR after NAC in breast cancer was 0.91 (95\% CI: 0.88, 0.93), which indicated good diagnostic performance. However, the homogeneity test of sensitivity and specificity showed notable heterogeneity. The threshold effect might be a source of heterogeneity, as most of the studies included in the present analysis set a threshold but did not pre-specify the threshold. The results of threshold effect assessment indicated that the threshold effect was indeed the most important factor and likely contributed to $95 \%$ of the heterogeneity.

In addition to the threshold effect, certain putative factors might enhance heterogeneity, for example the choice of the $b$ value may affect the ADC calculated by multiple pools diffusing at different rates [27]. The ADCs tend to be higher due to the contribution of perfusion in low $b$ values and may also be preferable for differentiating malignant from benign tissues exclusively based on their water diffusion characteristics at high $b$ values. However, the higher the $b$ value is, the worse the signal-to-noise ratio becomes; this relationship restricts the clinical application of a high $b$ value. To date, there is no consensus regarding the optimal b value in DWI studies [9]. In our subgroup analysis (Table 3 ), the results demonstrated that higher b value subgroups might outperform lower $b$ value subgroups in assessing the pCR to NAC, with a higher pooled specificity ( 0.85 vs. 0.76$)$ and a comparable pooled sensitivity ( 0.89 vs. 0.88 ). However, there were no notable differences between these two subgroups.

It has been shown that the change in diffusion coefficients is inversely correlated with therapeutic responses, and several studies [11-13, 18, 19, 23, 24] have noted that the change in ADC is an optimal biomarker for predicting the $\mathrm{pCR}$ in breast cancer. However, some 
studies $[15,21,25]$ have shown that the pre-NAC ADC value is higher in subjects achieving a pCR compared with those showing residual disease, while other studies $[17,20]$ have suggested using post-NAC ADC. Therefore, we performed a subgroup analysis of different biomarkers of DWI. The performance of the $\triangle \mathrm{ADC}$ subgroup appeared to be equivalent to that of the post-NAC ADC subgroup, with a comparable pooled sensitivity and pooled specificity. Moreover, both subgroups had a higher pooled specificity than did the pre-NAC ADC subgroup ( 0.80 vs. 0.63$)$. Thus, the breast cancer cells of responders are reduced and become necrotic in the form of a sieve during NAC, resulting in more significant changes in the diffusion parameters than observed for the breast cancer cells of non-responders.

Using a traditional monoexponential model, the ADC value can be calculated and used to quantitatively reflect the diffusion of water molecules in cancer tissue
[27]. However, both pure molecular diffusion and perfusion in microcapillary circulation contribute to the ADC value. This contribution weakens the ability of ADC to characterise tissue microstructure [25]. Therefore, an IVIM model was developed to separate molecular diffusion from perfusion by using a wide range of low and high b values [28]. Only 2 of the 15 studies included in our meta-analysis followed the IVIM model. Che et al. [14] demonstrated that the best biomarker was the change in the true molecular diffusion coefficient (D), which yielded a high sensitivity of 1.00 and a specificity of 0.79 . Bedair et al. [25] further suggested that the pre-treatment distributed diffusion coefficient (DDC) could potentially predict the $\mathrm{pCR}$ in breast cancer treatment with a sensitivity of 0.79 and a specificity of 0.73 . In our subgroup analysis, we found that the IVIM model subgroup appeared to be equivalent to the ADC model subgroup, with a similar pooled sensitivity ( 0.86 vs. 0.88$)$

\section{IORIS M \\ PRISMA 2009 Flow Diagram}

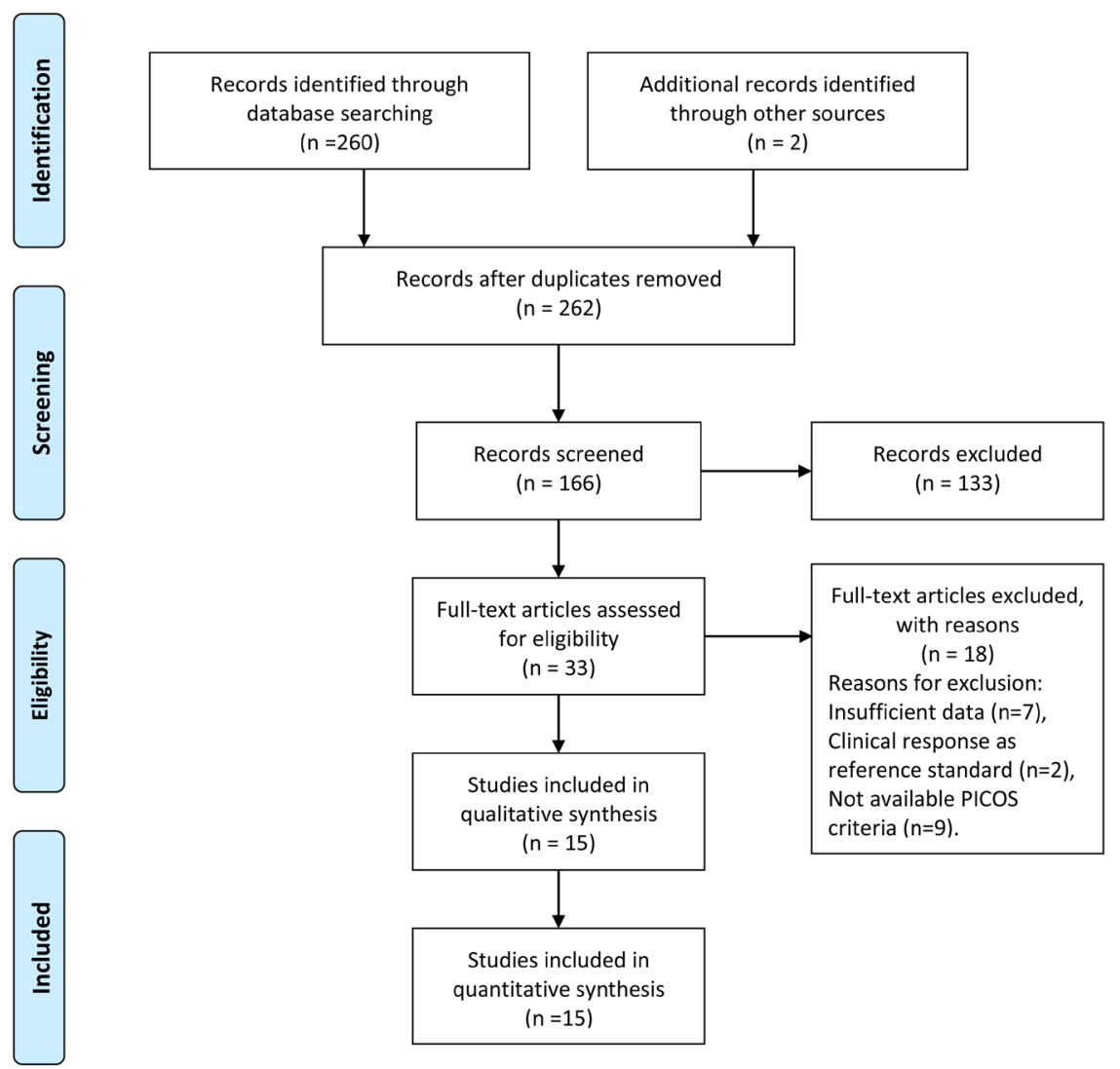

From: Moher D, Liberati A, Tetzlaff J, Altman DG, The PRISMA Group (2009). Preferred Reporting Items for Systematic Reviews and MetaAnalyses: The PRISMA Statement. PLoS Med 6(6): e1000097. doi:10.1371/journal.pmed1000097

For more information, visit www.prisma-statement.org.

Figure 1: Flowchart illustrating the selection of the studies. 
Table 2: Principal characteristics of the included studies

\begin{tabular}{|c|c|c|c|c|c|c|c|c|c|c|}
\hline Study & Year & Design & $\begin{array}{c}\text { Time of } \\
\text { scan }\end{array}$ & Field & Type & b value & $\begin{array}{c}\text { Evaluate } \\
\text { index }\end{array}$ & Cut-off & Sen & Spe \\
\hline An, Y & 2015 & Retro & $\begin{array}{c}\text { B/A(post } \\
\text { NAC) }\end{array}$ & $1.5 \mathrm{~T}$ & DWI & 0,750 & $\triangle \mathrm{ADC}$ & $15.2 \%$ & 0.67 & 0.71 \\
\hline Belli & 2011 & Pro & $\mathrm{B} / \mathrm{A}(\mathrm{NR})$ & $1.5 \mathrm{~T}$ & DWI & 0,1000 & $\triangle \mathrm{ADC}$ & $68.0 \%$ & 0.80 & 0.85 \\
\hline Bufi & 2014 & Retro & $\begin{array}{c}\mathrm{B} / \mathrm{A}(4-6 \\
\text { cycles })\end{array}$ & $1.5 \mathrm{~T}$ & DWI & 0,1000 & $\triangle \mathrm{ADC}$ & NR & 0.87 & 0.59 \\
\hline \multirow[t]{3}{*}{ Che } & 2016 & NR & $\begin{array}{c}\mathrm{B} / \mathrm{A}(2-3 \\
\text { cycles })\end{array}$ & $3.0 \mathrm{~T}$ & IVIM & 0,800 & $\Delta \mathrm{D}$ & $\Delta 0.163^{\mathrm{a}}$ & 1.00 & 0.79 \\
\hline & & & & & & & pre-D & $0.874^{\mathrm{a}}$ & 0.69 & 0.65 \\
\hline & & & & & & & post-D & $0.971^{\mathrm{a}}$ & 1.00 & 0.63 \\
\hline Fangberget & 2011 & Pro & $\begin{array}{l}\mathrm{B} / \mathrm{A}(4 \\
\text { cycles })\end{array}$ & $3.0 \mathrm{~T}$ & DWI & $100,250,800$ & pre-ADC & $1.420^{\mathrm{a}}$ & 0.91 & 0.81 \\
\hline \multirow[t]{3}{*}{$\mathrm{Li}$} & 2015 & Pro & $\begin{array}{l}\mathrm{B} / \mathrm{A}(1 \\
\text { cycles })\end{array}$ & $3.0 \mathrm{~T}$ & DWI & 0,600 & $\triangle \mathrm{ADC}$ & $5.5 \%$ & 0.50 & 0.76 \\
\hline & & & & & & & pre-ADC & $1.2^{\mathrm{a}}$ & 1.00 & 0.54 \\
\hline & & & & & & & post-ADC & $1.4^{\mathrm{a}}$ & 0.83 & 0.67 \\
\hline Liu & 2015 & Retro & $\begin{array}{c}\text { B/A(post } \\
\text { NAC) }\end{array}$ & $3.0 \mathrm{~T}$ & DWI & 0,800 & post-ADC & NR & 0.69 & 0.94 \\
\hline Luo & 2014 & NR & $\begin{array}{c}\text { B/A(post } \\
\text { NAC) }\end{array}$ & $3.0 \mathrm{~T}$ & DWI & 0,800 & $\triangle \mathrm{ADC}$ & $42.5 \%$ & 0.89 & 0.74 \\
\hline Park & 2010 & Retro & $\begin{array}{c}\text { B/A(post } \\
\text { NAC) }\end{array}$ & $1.5 \mathrm{~T}$ & DWI & 0,750 & post-ADC & $1.17^{\mathrm{a}}$ & 0.94 & 0.71 \\
\hline Park S & 2012 & Retro & $\begin{array}{c}\mathrm{B} / \mathrm{A}(3-6 \\
\text { cycles })\end{array}$ & $1.5 \mathrm{~T}$ & DWI & 0,750 & $\triangle \mathrm{ADC}$ & $54.9 \%$ & 1.00 & 0.70 \\
\hline Richard $\dagger$ & 2013 & Retro & $\begin{array}{c}\text { B/A(post } \\
\text { NAC) }\end{array}$ & $1.5 \mathrm{~T}$ & DWI & 50,700 & pre-ADC & $1.29^{\mathrm{a}}$ & 1.00 & 0.38 \\
\hline \multirow[t]{3}{*}{ Shin } & 2012 & Retro & $\begin{array}{c}\text { B/A(post } \\
\text { NAC) }\end{array}$ & $1.5 \mathrm{~T}$ & DWI & $\begin{array}{l}100,500 \\
800,1000\end{array}$ & $\triangle \mathrm{ADC}$ & $40.7 \%$ & 1.00 & 0.91 \\
\hline & & & & & & & pre-ADC & $0.92^{\mathrm{a}}$ & 0.80 & 0.65 \\
\hline & & & & & & & post-ADC & $1.19^{\mathrm{a}}$ & 1.00 & 0.70 \\
\hline Weis & 2015 & Retro & $\begin{array}{l}\mathrm{B} / \mathrm{A}(1 \\
\text { cycles) }\end{array}$ & $3.0 \mathrm{~T}$ & DWI & $0,500 / 600$ & $\triangle \mathrm{ADC}$ & NR & 0.92 & 0.84 \\
\hline Woodhams & 2010 & NR & $\begin{array}{l}\mathrm{B} / \mathrm{A}(4 \\
\text { cycles })\end{array}$ & $1.5 \mathrm{~T}$ & DWI & 0,1500 & $\triangle \mathrm{ADC}$ & $\begin{array}{c}\text { Category } \\
1^{\mathrm{b}}\end{array}$ & 0.97 & 0.89 \\
\hline \multirow[t]{2}{*}{ Bedair } & 2017 & Pro & $\begin{array}{l}\mathrm{B} / \mathrm{A}(3 \\
\text { cycles })\end{array}$ & $3.0 \mathrm{~T}$ & IVIM & $\begin{array}{c}0,60,120,300 \\
600,900\end{array}$ & DDC & $1.14^{\mathrm{a}}$ & 0.79 & 0.73 \\
\hline & & & & & & & ADC & $1.01^{\mathrm{a}}$ & 0.79 & 0.67 \\
\hline
\end{tabular}

Note: ${ }^{\text {a }}$, ADC or D or DDC $\left(\times 10^{-3} \mathrm{~mm}^{2} / \mathrm{s}\right)$; ${ }^{\text {b }}$, DWI was classified into 4 categories: category 1 indicated no residual disease. $\dagger$, the analysis was available in the triple-negative subtype.

Abbreviations: ADC, apparent diffusion coefficient; $\triangle \mathrm{ADC}$, change in apparent diffusion coefficient; $\mathrm{B} / \mathrm{A}$, before NAC and after NAC; D, true molecular diffusion coefficient; DDC, distributed diffusion coefficient; $\Delta \mathrm{D}$, change in true molecular diffusion coefficient; IVIM, intravoxel incoherent motion; Pro, prospective; Retro, retrospective; NR, not reported; Sen, sensitivity; Spe, specificity.

and pooled specificity ( 0.76 vs. 0.79$)$. Based on the small number and sample size of the studies, additional clinical trials focused on the ability IVIM to predict the pCR in breast cancer treatment are required.
Classically, the response to NAC has been identified by CE-MRI alone with Response Evaluation Criteria in Solid Tumours (RECIST) during NAC [29]. Compared with CE-MRI, DWI is able to obtain both anatomic and 
functional information simultaneously. Two previous meta-analyses [30, 31] compared the accuracy of DWI and CE-MRI for indirectly evaluating the pCR to breast cancer NAC, with only 6 [30] and 8 [31] studies available. Both meta-analyses congruously reported that DWI had a higher pooled sensitivity but a lower pooled specificity than CEMRI. As direct comparisons provide the best effects of the diagnostic test accuracy of the two techniques [32, 33], we included exclusively head-to-head comparative studies that evaluated these two techniques in the same cohort. Our results showed that the pooled sensitivity, specificity and AUC of DWI were slightly higher than those of CE-
MRI, in contrast to the results of the previous studies. Based on the data of the DOR value shown in Table 4, we observed an underlying trend that DWI is increasingly superior to CE-MRI with an increase in the number of DWI studies.

Some intrinsic limitations should be considered in the present study. First, because different tumour subtypes of breast cancer receive different NAC regimens with different histopathological responses, a quantitative analysis based on tumour subtype is highly desirable. However, only two studies [17, 21] on breast cancer subtypes were identified in the present meta-analysis.

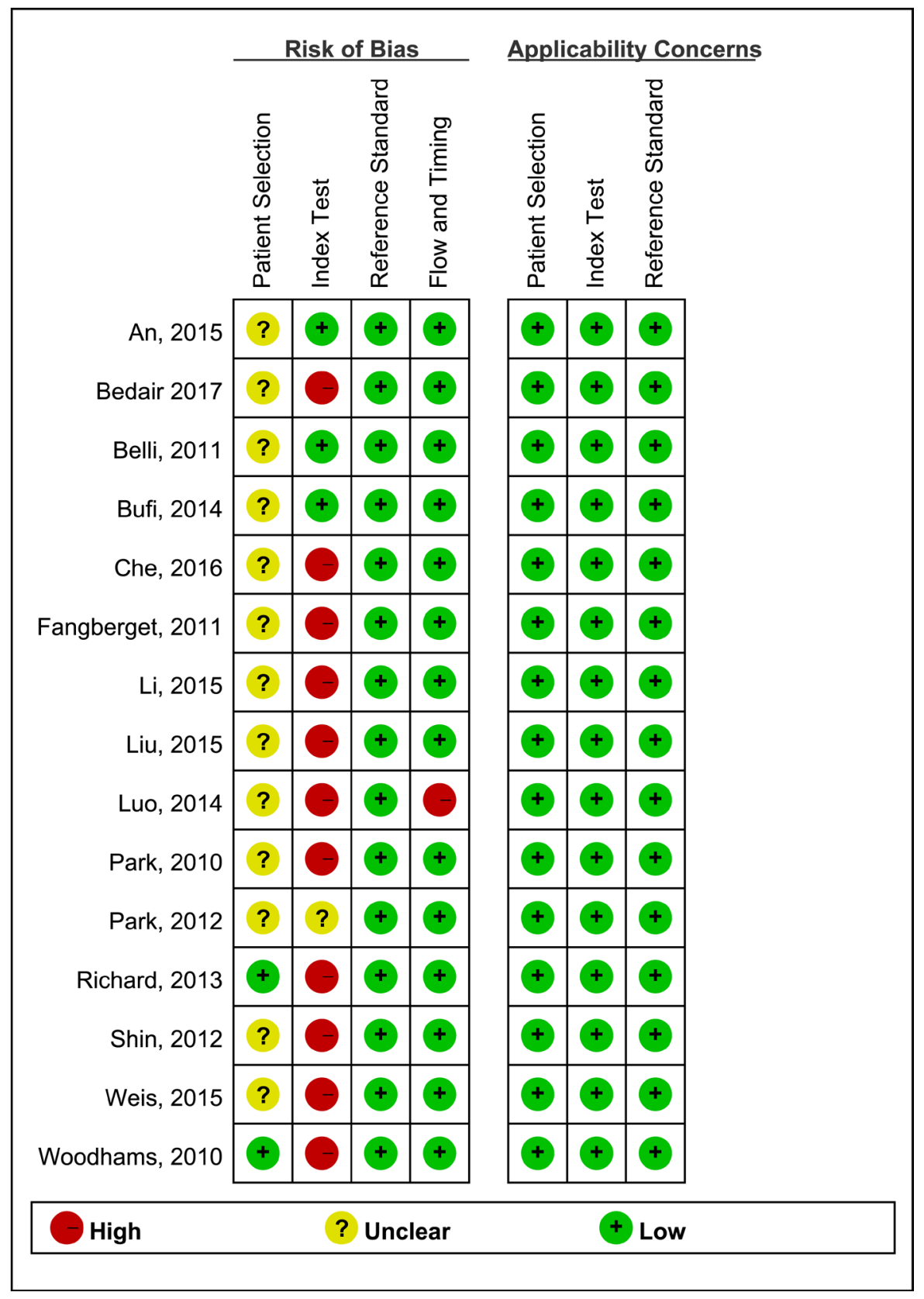

Figure 2: Risk of bias and applicability concerns summary of the 15 included studies. 
Table 3: Sensitivity and specificity estimates for each subgroup

\begin{tabular}{|c|c|c|c|c|c|}
\hline Subgroup & No. of studies & Mean SEN (\%) & Mean SPE (\%) & DOR & AUC (\%) \\
\hline \multicolumn{6}{|l|}{$\mathrm{b}$ value $\left(\mathrm{s} / \mathrm{m}^{2}\right)$} \\
\hline$\geq 1000$ & 5 & $89(79-95)$ & 85 (69-93) & $45(13-160)$ & $91(89-94)$ \\
\hline$<1000$ & 10 & 88 (79-93) & $76(64-85)$ & $22(12-41)$ & $90(87-92)$ \\
\hline \multicolumn{6}{|l|}{ Biomarker } \\
\hline$\triangle \mathrm{ADC}$ & 10 & $88(75-94)$ & $80(71-87)$ & $29(10-83)$ & $91(88-93)$ \\
\hline Pre-NAC ADC & 6 & $90(74-96)$ & $63(52-73)$ & $15(5-41)$ & $79(75-82)$ \\
\hline Post-NAC ADC & 5 & $91(78-96)$ & $78(58-90)$ & 34 (13-87) & $92(90-95)$ \\
\hline \multicolumn{6}{|l|}{ Study design } \\
\hline Retrospective & 8 & $91(80-96)$ & $75(60-86)$ & $30(12-77)$ & 92 (89-94) \\
\hline Prospective & 4 & $82(71-84)$ & $76(66-84)$ & $15(7-34)$ & $86(83-89)$ \\
\hline \multicolumn{6}{|l|}{ Magnetic field } \\
\hline $1.5 \mathrm{~T}$ & 9 & $91(83-95)$ & 77 (63-87) & $33(13-80)$ & $93(90-95)$ \\
\hline $3.0 \mathrm{~T}$ & 6 & $85(70-91)$ & $82(71-89)$ & $22(10-47)$ & $89(86-91)$ \\
\hline \multicolumn{6}{|l|}{ Model } \\
\hline $\mathrm{ADC}$ & 13 & 88 (81-93) & 79 (69-87) & $27(15-50)$ & $91(88-93)$ \\
\hline IVIM & 2 & $86(64-97)$ & $76(63-87)$ & $14(3-60)$ & NA \\
\hline
\end{tabular}

Note: Numbers in parentheses are the $95 \%$ CIs. NA $=$ not applicable.

Liu et al. [17] showed that post-NAC ADC appears to be a promising tool for determining the $\mathrm{pCR}$ to NAC in breast cancer subtypes. The AUCs of the luminal A, luminal B, HER2-enriched, and triple-negative subtypes were $0.86,0.86,0.79$, and 0.75 , respectively. However, Richard et al. [21] found that the pre-NAC ADC of the triple-negative subtype was significantly higher in nonresponders than in the pCR group, but no significant
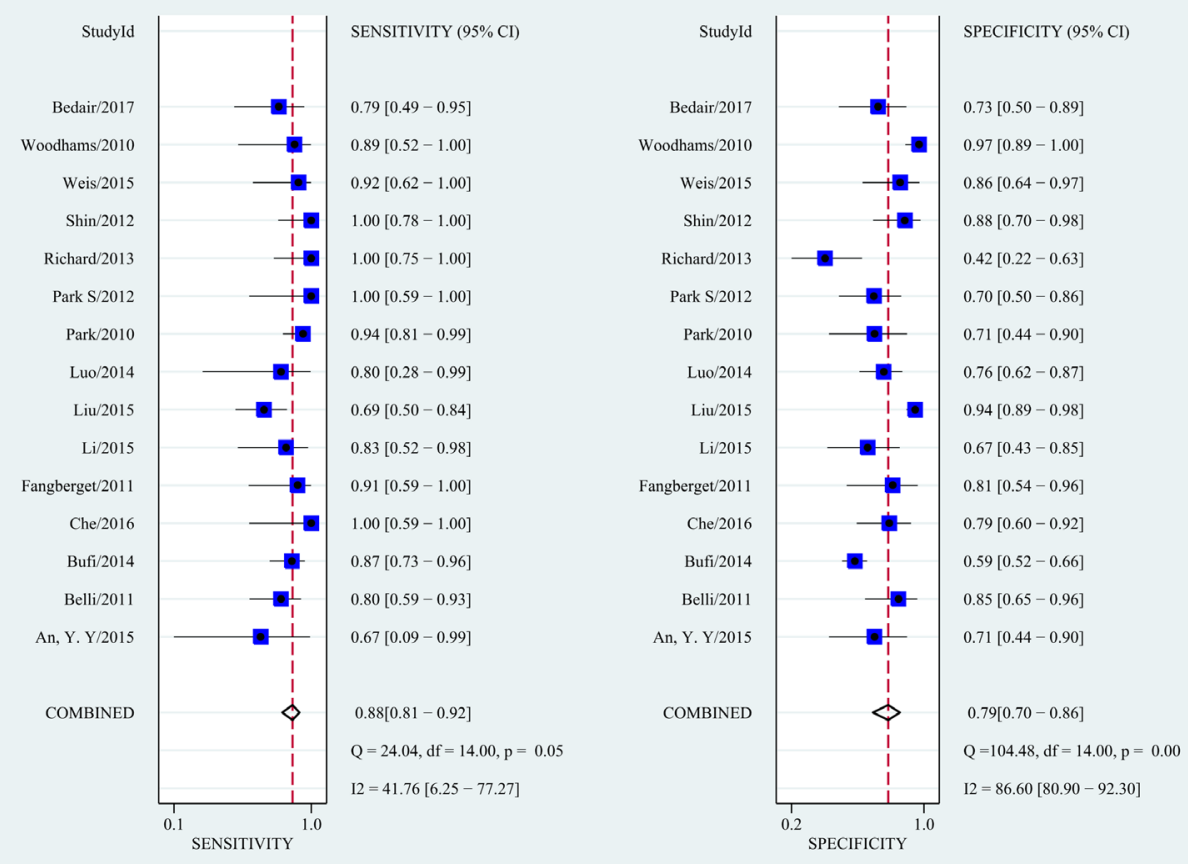

Figure 3: Forest plots of the SEN and SPE and corresponding 95\% CIs for DWI as an assessor of the pathologic response to NAC. 
Table 4: Summary of meta-analyses focused on CE-MRI and DWI for the assessment of breast cancer responses to $\mathrm{NAC}$

\begin{tabular}{|c|c|c|c|c|c|c|c|c|c|}
\hline Study & Search date & Comparative & No. & Technique & $\operatorname{PSEN}(95 \% \mathrm{CI})$ & $\operatorname{PSPE}(95 \% \mathrm{CI})$ & $\operatorname{DOR}(95 \% \mathrm{CI})$ & rDOR & AUC (95\% CI) \\
\hline \multirow[t]{2}{*}{$\mathrm{Wu}[30]$} & $\begin{array}{l}2000 \text { to } \\
2012\end{array}$ & $\begin{array}{c}\text { Indirect } \\
\text { comparative }\end{array}$ & 30 & CE-MRI & $0.68(0.57,0.77)$ & $0.91(0.87,0.94)$ & $55.59(21.80,141.80)$ & & NR \\
\hline & & & 6 & DWI & $0.93(0.82,0.97)$ & $0.79(0.74,0.83)$ & $20.99(13.24,33.25)$ & 0.38 & NR \\
\hline \multirow[t]{2}{*}{ Liu [31] } & 1992-2015 & $\begin{array}{c}\text { Indirect } \\
\text { comparative }\end{array}$ & 54 & CE-MRI & $0.68(0.66,0.78)$ & $0.84(0.80,0.88)$ & $13.82(7.28,26.23)$ & & $0.88(\mathrm{NR})$ \\
\hline & & & 8 & DWI & $0.79(0.68,0.88)$ & $0.75(0.70,0.80)$ & $18.68(6.88-50.73$ & 1.35 & $0.87(\mathrm{NR})$ \\
\hline \multirow[t]{2}{*}{ Our } & $\begin{array}{c}2000 \text { to } \\
2017\end{array}$ & Direct comparative & 9 & CE-MRI & $0.84(0.74,0.91)$ & $0.76(0.64,0.85)$ & $16.57(9.80,28.02)$ & & $0.88(0.84,0.90)$ \\
\hline & & & 9 & DWI & $0.89(0.81,0.93)$ & $0.81(0.71,0.89)$ & $33.72(13.93,81.59)$ & 2.04 & $0.91(0.88,0.93)$ \\
\hline
\end{tabular}

Notes: No.= no. of studies; PSEN = pooled sensitivities; PSPE = pooled specificities; DOR = diagnostic odds ratio; AUC= areas under the ROC curve; DWI = diffusion weighted imaging; CE-MRI = contrast-enhanced MRI; rDOR = the ratio of DOR value of DWI divided by DOR value of CE-MRI; NR = not reported.

differences were observed in the luminal $\mathrm{A}$ and $\mathrm{B}$ subtypes. Second, diagnostic test accuracy estimates could also be influenced by the definition of pCR [34]. As there were too many pCR definitions (e.g., Chevallier-Sataloff classification, Miller-Payne Grading System, Mandard's TRG classification, RCB Index, or classification by user-defined) to perform a subgroup analysis, we did not assess pCR in our analyses. Third, there is notable heterogeneity in this meta-analysis. Many other factors, such as standards of DWI measurement, analysis, and cutoff values of diagnosis in DWI techniques, should be investigated. However, no consensus has been reached regarding those standards, making it difficult to summarise these factors in a meta-analysis.

In summary, this meta-analysis, which included 15 studies and 1081 patients, showed that DWI may be an accurate and nonradioactive imaging technique and might even be superior to conventional CE-MRI with respect to identifying the pCR to NAC in breast cancer. However, considering the notable heterogeneity and existing inherent

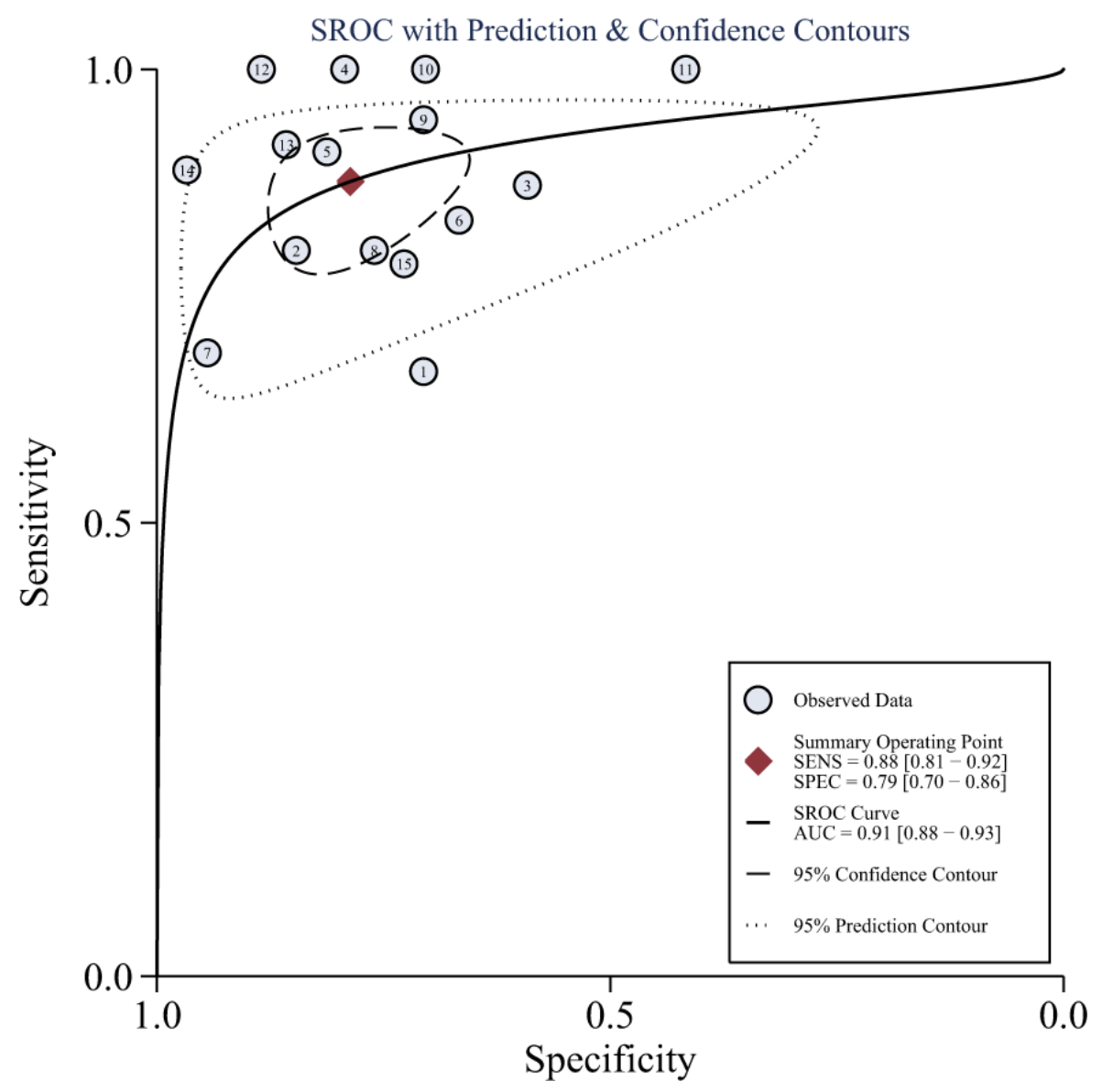

Figure 4: Hierarchical summary receiver operating characteristic (HSROC) curves from the bivariate model of DWI. 


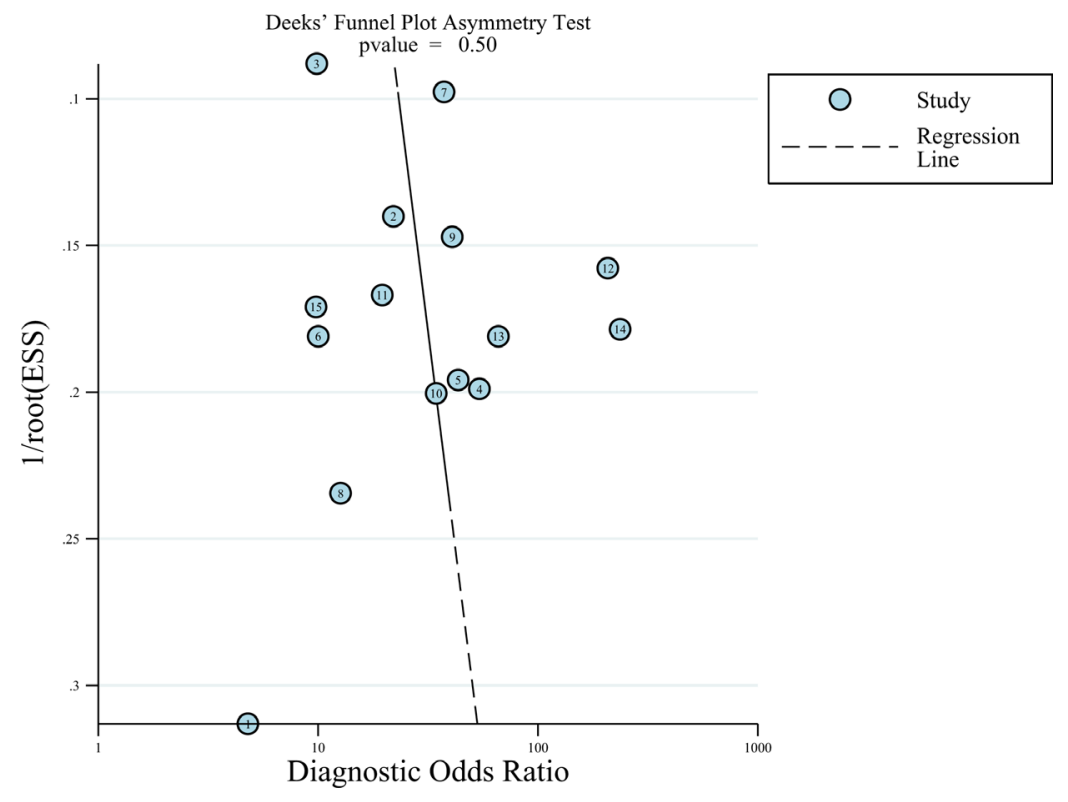

Figure 5: Funnel plot of publication bias. The results showed no evidence of notable publication bias $(P=0.50)$.

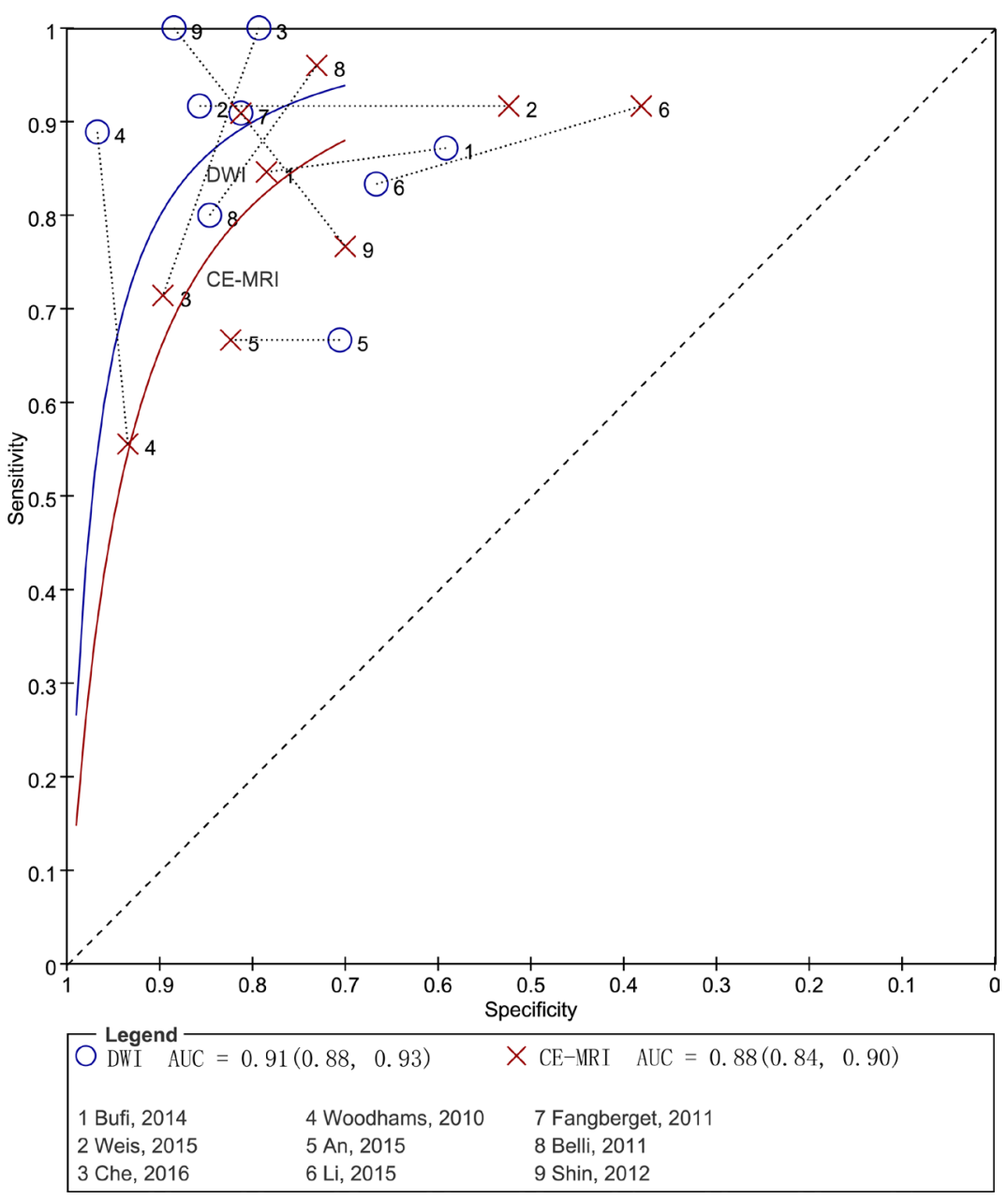

Figure 6: Pairs of observed sensitivity and specificity values for DWI and CE-MRI in HSROC curves. 
limitations, this technique should be applied cautiously. In addition, there are a variety of issues concerning the assessment of DWI techniques for estimating breast cancer responses to NAC. Therefore, using a clear pCR definition, appropriate standards of DWI measurement, analysis, cutoff values, and large scale/multi-centred and well-designed clinical trials are necessary to assess the diagnostic value of DWI in breast NAC.

\section{MATERIALS AND METHODS}

We employed the Preferred Reporting Items for Systematic Reviews and Meta-Analyses statement [35] to enhance the reporting of the present study (Figure 1).

\section{Search strategy}

A structured approach was followed to detect the patient population, interventions, comparators, outcomes, and study design (PICOS criteria) [35]. Two authors searched the data sources (PUBMED, EMBASE, Web of Science, and the Cochrane Library) independently. The search strategy (Appendix A) comprised both subject headings (MeSH terms) and keywords for the target condition (breast cancer), the imaging under investigation (DWI), and the interventions (neoadjuvant therapy). We limited our search to studies published no later than July 2017. Review articles, letters, comments, case reports, and unpublished articles were excluded. Extensive crosschecking of the references in all the retrieved articles was performed.

\section{Criteria for inclusion in the study}

Studies were considered available if the following PICOS criteria were met: (a) the patient population consisted of primary breast cancer confirmed histologically, (b) the imaging response to NAC was assessed using DWI, (c) histopathologic analysis was eligible as a gold standard, (d) a pCR or a near-pCR to NAC was described as an outcome and (e) both prospective and retrospective design were included.

We excluded studies if a $2 \times 2$ table could not be extracted from the data, if a full-text translation or evaluation for Non-English and non-Chinese articles could not be obtained, and if multiple reports were published for the same cohort. In the latter case, the most detailed or recent publication was extracted.

\section{Selection of articles}

The selection of articles was performed independently by two authors, who initially screened the search results in titles and abstracts and further retrieved the full text of all potentially relevant reports. Next, the authors reviewed all relevant items according to the predefined inclusion criteria. Disagreements were arbitrated by a third author, who assessed all involved issues.

\section{Quality assessment and data extraction}

For each included study, the methodological quality was evaluated independently by the three aforementioned authors, who extracted data from the selected reports using the standard quality assessment of diagnostic studies (QUADAS-2) items [36-38]. Additionally, associated data, including author, study nation, population and tumour characteristics, descriptions of definition of pCR and NAC regimens, study design, magnetic field strength, standards of DWI techniques, evaluation time, and descriptions of interpretations of the diagnostic tests, were also extracted from each study. The true-positive, false-positive, true-negative, and falsenegative data were extracted and derived to construct $2 \times 2$ contingency tables.

\section{Meta-analysis}

We constructed forest plots to demonstrate the variations of the sensitivity (SEN) and specificity (SPE) estimates together for DWI in each study and calculated the SEN, SPE, PLR, NLR and DOR values with 95\% CIs. Hierarchical summary receiver operating characteristic (HSROC) curves were generated to assess SEN and SPE [39]. Standard $\chi^{2}$-testing and the inconsistency index (I-squared, $\mathrm{I}^{2}$ ) were used to estimate the heterogeneity of the individual studies using Stata software (version 14.0, Stata Corporation, College Station, TX, USA). If notable heterogeneities were detected $\left(\mathrm{P}<0.1\right.$ or $\mathrm{I}^{2}>50 \%$ [40]), the performance was pooled using a random-effects coefficient binary regression model; otherwise, a fixedeffects coefficient binary regression model was used [32]. Threshold effect testing, sensitivity analysis and metaregression were used to explore heterogeneity.

The following subgroup analyses were carried out: (a) comparisons of studies using different b values: lower $b$ value subgroup $\left(\geq 1000 \mathrm{~s} / \mathrm{m}^{2}\right)$ or higher $b$ value subgroup $\left(<1000 \mathrm{~s} / \mathrm{m}^{2}\right)$; (b) comparisons of studies with different biomarkers: $\triangle \mathrm{ADC}$ subgroup, pre-treatment subgroup or post-treatment subgroup; (c) comparisons of studies using a different magnetic field: $1.5 \mathrm{~T}$ subgroup or $3.0 \mathrm{~T}$ subgroup; (d) comparisons of studies with a different study design: retrospective subgroup or prospective subgroup; and (e) comparisons of studies using different diffusion models: ADC subgroup or IVIM subgroup.

Deeks funnel plots were generated and an asymmetry test was performed to assess publication bias. The existence of a nonzero slope coefficient $(P<0.05)$ was considered evidence of notable publication bias [41]. 


\section{Abbreviations}

AUC: area under the ROC curve; ADC: apparent diffusion coefficient; CE: contrast enhanced; CI: confidence interval; DOR: diagnostic odds ratio; DWI: diffusion-weighted imaging ; HSROC: hierarchical summary receiver operating characteristic; IVIM: intravoxel incoherent motion; MRI: magnetic resonance imaging; NAC: neoadjuvant chemotherapy; NLR: negative likelihood ratio; pCR: pathologic complete response; PLR: positive likelihood ratio; QUADAS: quality assessment of diagnostic studies; SEN: sensitivity; SPE: specificity.

\section{Author contributions}

Chen Lihua and Huang Xuequan contributed to the conception and design of the study and the editing of the manuscript; Chu Wei and Jin Weiwei contributed to data acquisition, the analysis and interpretation of the data, and the editing of the manuscript; and Liu Daihong, Wang Jian, and Geng Chengjun contributed to the statistical analysis.

\section{CONFLICTS OF INTEREST}

The authors declare no competing financial interests.

\section{FUNDING}

This study was supported by Wuxi Young Medical Talents (QNRC061).

\section{REFERENCES}

1. Goldhirsch A, Glick JH, Gelber RD, Coates AS, Senn HJ. Meeting highlights: International Consensus Panel on the Treatment of Primary Breast Cancer. Seventh International Conference on Adjuvant Therapy of Primary Breast Cancer. J Clin Oncol. 2001; 19:3817-27.

2. von Minckwitz G, Rezai M, Loibl S, Fasching PA, Huober J, Tesch H, Bauerfeind I, Hilfrich J, Eidtmann H, Gerber B, Hanusch C, Kuhn T, du Bois A, et al. Capecitabine in addition to anthracycline- and taxane-based neoadjuvant treatment in patients with primary breast cancer: phase III GeparQuattro study. J Clin Oncol. 2010; 28:2015-23.

3. Thomas E, Holmes FA, Smith TL, Buzdar AU, Frye DK, Fraschini G, Singletary SE, Theriault RL, McNeese MD, Ames F, Walters R, Hortobagyi GN. The use of alternate, non-cross-resistant adjuvant chemotherapy on the basis of pathologic response to a neoadjuvant doxorubicin-based regimen in women with operable breast cancer: long-term results from a prospective randomized trial. J Clin Oncol. 2004; 22:2294-302.
4. Yankeelov TE, Atuegwu N, Hormuth D, Weis JA, Barnes SL, Miga MI, Rericha EC, Quaranta V. Clinically relevant modeling of tumor growth and treatment response. Sci Trans1 Med. 2013; 5:187ps9.

5. Lee CI, Gold LS, Nelson HD, Chou R, Ramsey SD, Sullivan SD. Comparative effectiveness of imaging modalities to determine metastatic breast cancer treatment response. Breast. 2015; 24:3-11.

6. Choi JH, Lim HI, Lee SK, Kim WW, Kim SM, Cho E, Ko EY, Han BK, Park YH, Ahn JS, Im YH, Lee JE, Yang $\mathrm{JH}$, et al. The role of PET CT to evaluate the response to neoadjuvant chemotherapy in advanced breast cancer: comparison with ultrasonography and magnetic resonance imaging. J Surg Oncol. 2010; 102:392-7.

7. Abdel Razek AA, Gaballa G, Denewer A, Tawakol I. Diffusion weighted MR imaging of the breast. Acad Radiol. 2010; 17:382-6.

8. Hatakenaka M, Soeda H, Yabuuchi H, Matsuo Y, Kamitani T, Oda Y, Tsuneyoshi M, Honda H. Apparent diffusion coefficients of breast tumors: clinical application. Magn Reson Med Sci. 2008; 7:23-9.

9. Chen L, Liu M, Bao J, Xia Y, Zhang J, Zhang L, Huang $\mathrm{X}$, Wang J. The correlation between apparent diffusion coefficient and tumor cellularity in patients: a metaanalysis. PLoS One. 2013; 8:e79008.

10. Woodhams R, Kakita S, Hata H, Iwabuchi K, Umeoka S, Mountford CE, Hatabu H. Diffusion-weighted imaging of mucinous carcinoma of the breast: evaluation of apparent diffusion coefficient and signal intensity in correlation with histologic findings. AJR Am J Roentgenol. 2009; 193:260-6.

11. An YY, Kim SH. Treatment Response Evaluation of Breast Cancer after Neoadjuvant Chemotherapy and Usefulness of the Imaging Parameters of MRI and PET/CT. J Korean Med Sci. 2015; 30:808-15.

12. Belli P, Costantini M, Ierardi C, Bufi E, Amato D, Mule A, Nardone L, Terribile D, Bonomo L. Diffusion-weighted imaging in evaluating the response to neoadjuvant breast cancer treatment. Breast J. 2011; 17:610-9.

13. Bufi E, Belli P, Di Matteo M, Terribile D, Franceschini G, Nardone L, Petrone G, Bonomo L. Effect of breast cancer phenotype on diagnostic performance of MRI in the prediction to response to neoadjuvant treatment. Eur $\mathrm{J}$ Radiol. 2014; 83:1631-8.

14. Che S, Zhao X, Ou Y, Li J, Wang M, Wu B, Zhou C. Role of the Intravoxel Incoherent Motion Diffusion Weighted Imaging in the Pre-treatment Prediction and Early Response Monitoring to Neoadjuvant Chemotherapy in Locally Advanced Breast Cancer. Medicine (Baltimore). 2016; 95:e2420.

15. Fangberget A, Nilsen LB, Hole KH, Holmen MM, Engebraaten O, Naume B, Smith HJ, Olsen DR, Seierstad T. Neoadjuvant chemotherapy in breast cancer-response evaluation and prediction of response to treatment using 
dynamic contrast-enhanced and diffusion-weighted MR imaging. Eur Radiol. 2011; 21:1188-99.

16. Li X, Abramson RG, Arlinghaus LR, Kang H, Chakravarthy AB, Abramson VG, Farley J, Mayer IA, Kelley MC, Meszoely IM, Means-Powell J, Grau AM, Sanders M, et al. Multiparametric magnetic resonance imaging for predicting pathological response after the first cycle of neoadjuvant chemotherapy in breast cancer. Invest Radiol. 2015; 50:195-204.

17. Liu S, Ren R, Chen Z, Wang Y, Fan T, Li C, Zhang P. Diffusion-weighted imaging in assessing pathological response of tumor in breast cancer subtype to neoadjuvant chemotherapy. J Magn Reson Imaging. 2015; 42:779-87.

18. Luo Y, Yu J, Xu Z, Zeng H, Chen H. [Evaluation of pathologic response of breast cancer to neoadjuvant chemotherapy with magnetic resonance diffusion weighted imaging]. [Article in Chinese]. Sheng Wu Yi Xue Gong Cheng Xue Za Zhi. 2014; 31:1336-41.

19. Park SH, Moon WK, Cho N, Chang JM, Im SA, Park IA, Kang KW, Han W, Noh DY. Comparison of diffusion-weighted MR imaging and FDG PET/CT to predict pathological complete response to neoadjuvant chemotherapy in patients with breast cancer. Eur Radiol. 2012; 22:18-25.

20. Park SH, Moon WK, Cho N, Song IC, Chang JM, Park IA, Han W, Noh DY. Diffusion-weighted MR imaging: pretreatment prediction of response to neoadjuvant chemotherapy in patients with breast cancer. Radiology. 2010; 257:56-63.

21. Richard R, Thomassin I, Chapellier M, Scemama A, de Cremoux P, Varna M, Giacchetti S, Espie M, de Kerviler E, de Bazelaire C. Diffusion-weighted MRI in pretreatment prediction of response to neoadjuvant chemotherapy in patients with breast cancer. Eur Radiol. 2013; 23:2420-31.

22. Shin HJ, Baek HM, Ahn JH, Baek S, Kim H, Cha JH, Kim HH. Prediction of pathologic response to neoadjuvant chemotherapy in patients with breast cancer using diffusion-weighted imaging and MRS. NMR Biomed. 2012; 25:1349-59.

23. Weis JA, Miga MI, Arlinghaus LR, Li X, Abramson V, Chakravarthy AB, Pendyala P, Yankeelov TE. Predicting the Response of Breast Cancer to Neoadjuvant Therapy Using a Mechanically Coupled Reaction-Diffusion Model. Cancer Res. 2015; 75:4697-707.

24. Woodhams R, Kakita S, Hata H, Iwabuchi K, Kuranami M, Gautam S, Hatabu H, Kan S, Mountford C. Identification of residual breast carcinoma following neoadjuvant chemotherapy: diffusion-weighted imaging--comparison with contrast-enhanced MR imaging and pathologic findings. Radiology. 2010; 254:357-66.

25. Bedair R, Priest AN, Patterson AJ, McLean MA, Graves MJ, Manavaki R, Gill AB, Abeyakoon O, Griffiths JR, Gilbert FJ. Assessment of early treatment response to neoadjuvant chemotherapy in breast cancer using non-mono-exponential diffusion models: a feasibility study comparing the baseline and mid-treatment MRI examinations. Eur Radiol. 2017; 27:2726-36.

26. Leong KM, Lau P, Ramadan S. Utilisation of MR spectroscopy and diffusion weighted imaging in predicting and monitoring of breast cancer response to chemotherapy. J Med Imaging Radiat Oncol. 2015; 59:268-77.

27. Partridge SC, McDonald ES. Diffusion weighted magnetic resonance imaging of the breast: protocol optimization, interpretation, and clinical applications. Magn Reson Imaging Clin N Am. 2013; 21:601-24.

28. Cho GY, Gennaro L, Sutton EJ, Zabor EC, Zhang Z, Giri D, Moy L, Sodickson DK, Morris EA, Sigmund EE, Thakur SB. Intravoxel incoherent motion (IVIM) histogram biomarkers for prediction of neoadjuvant treatment response in breast cancer patients. Eur J Radiol Open. 2017; 4:101-7.

29. Marinovich ML, Macaskill P, Irwig L, Sardanelli F, von Minckwitz G, Mamounas E, Brennan M, Ciatto S, Houssami N. Meta-analysis of agreement between MRI and pathologic breast tumour size after neoadjuvant chemotherapy. Br J Cancer. 2013; 109:1528-36.

30. Wu LM, Hu JN, Gu HY, Hua J, Chen J, Xu JR. Can diffusion-weighted MR imaging and contrast-enhanced MR imaging precisely evaluate and predict pathological response to neoadjuvant chemotherapy in patients with breast cancer? Breast Cancer Res Treat. 2012; 135:17-28.

31. Gu YL, Pan SM, Ren J, Yang ZX, Jiang GQ. Role of Magnetic Resonance Imaging in Detection of Pathologic Complete Remission in Breast Cancer Patients Treated With Neoadjuvant Chemotherapy: A Meta-analysis. Clin Breast Cancer. 2017; 17:245-55.

32. Leeflang MM, Deeks JJ, Gatsonis C, Bossuyt PM. Systematic reviews of diagnostic test accuracy. Ann Intern Med. 2008; 149:889-97.

33. Brazzelli M, Sandercock PA, Chappell FM, Celani MG, Righetti E, Arestis N, Wardlaw JM, Deeks JJ. Magnetic resonance imaging versus computed tomography for detection of acute vascular lesions in patients presenting with stroke symptoms. Cochrane Database Syst Rev. 2009: CD007424.

34. Marinovich ML, Houssami N, Macaskill P, Sardanelli F, Irwig L, Mamounas EP, von Minckwitz G, Brennan ME, Ciatto S. Meta-analysis of magnetic resonance imaging in detecting residual breast cancer after neoadjuvant therapy. J Natl Cancer Inst. 2013; 105:321-33.

35. Moher D, Liberati A, Tetzlaff J, Altman DG. Preferred reporting items for systematic reviews and meta-analyses: the PRISMA statement. PLoS Med. 2009; 6:e1000097.

36. Whiting P, Rutjes AW, Reitsma JB, Bossuyt PM, Kleijnen J. The development of QUADAS: a tool for the quality assessment of studies of diagnostic accuracy included in systematic reviews. BMC Med Res Methodol. 2003; 3:25.

37. Whiting PF, Weswood ME, Rutjes AW, Reitsma JB, Bossuyt PN, Kleijnen J. Evaluation of QUADAS, a tool for the quality assessment of diagnostic accuracy studies. BMC Med Res Methodol. 2006; 6:9. 
38. Whiting PF, Rutjes AW, Westwood ME, Mallett S, Deeks JJ, Reitsma JB, Leeflang MM, Sterne JA, Bossuyt PM. QUADAS-2: a revised tool for the quality assessment of diagnostic accuracy studies. Ann Intern Med. 2011; 155:529-36.

39. Menke J. Bivariate random-effects meta-analysis of sensitivity and specificity with SAS PROC GLIMMIX. Methods Inf Med. 2010; 49:54-62, 62-4.
40. Higgins JP, Thompson SG, Deeks JJ, Altman DG. Measuring inconsistency in meta-analyses. BMJ. 2003; 327:557-60.

41. Deeks JJ, Macaskill P, Irwig L. The performance of tests of publication bias and other sample size effects in systematic reviews of diagnostic test accuracy was assessed. J Clin Epidemiol. 2005; 58:882-93. 